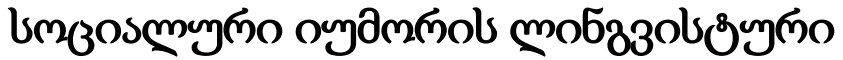 zss\&mgaolssonols
}

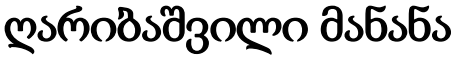

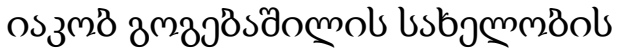

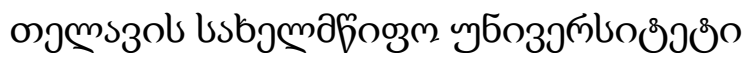

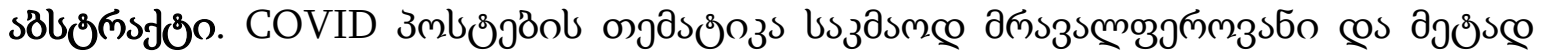

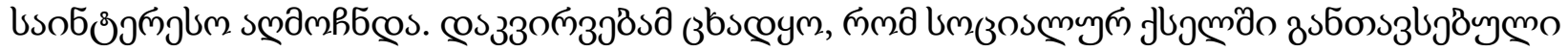

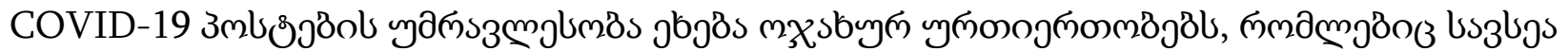

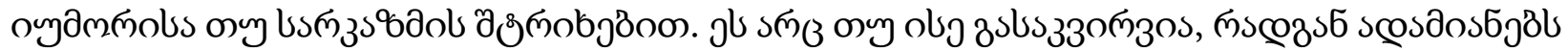

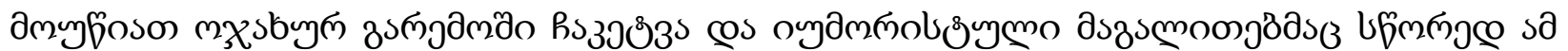

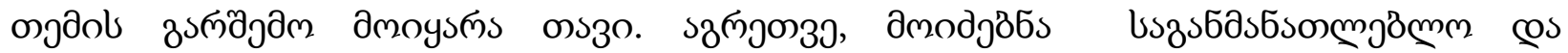

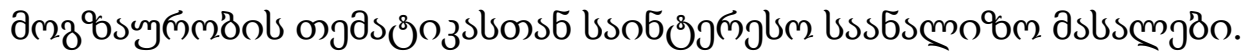

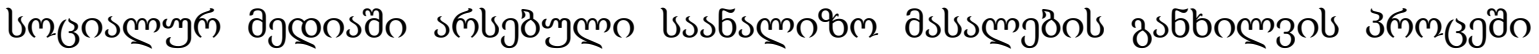

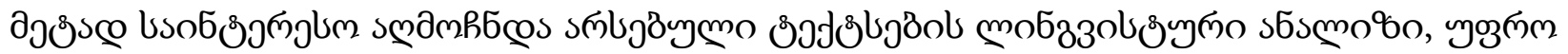

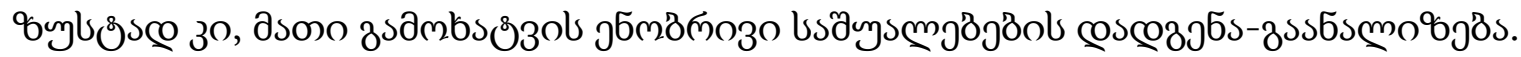

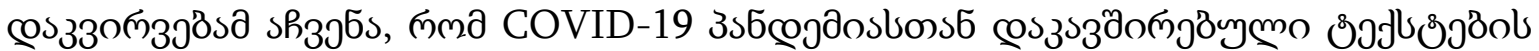

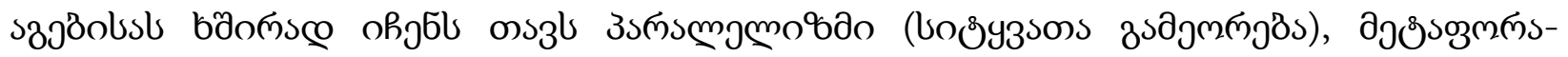

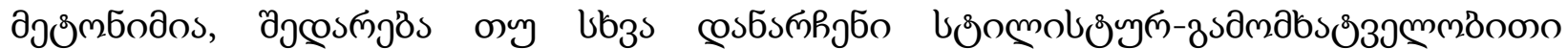

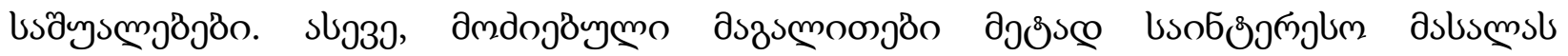

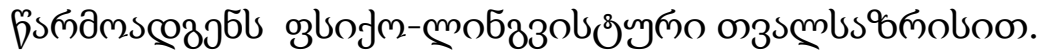

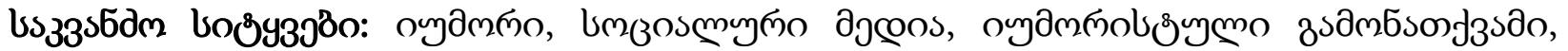
facebook, змауббозьъо

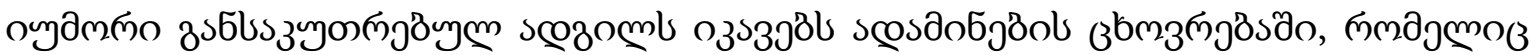

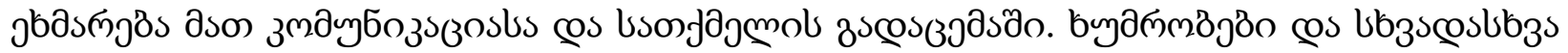

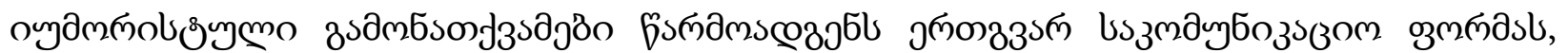

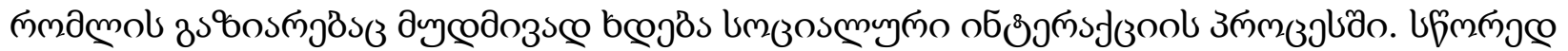

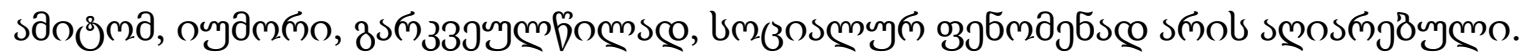

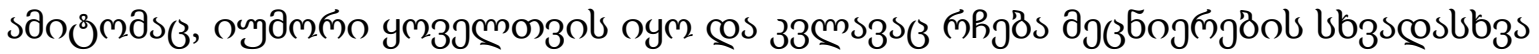

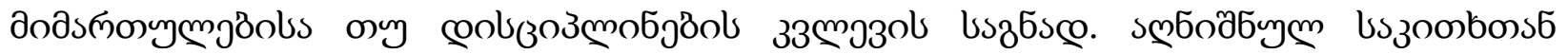

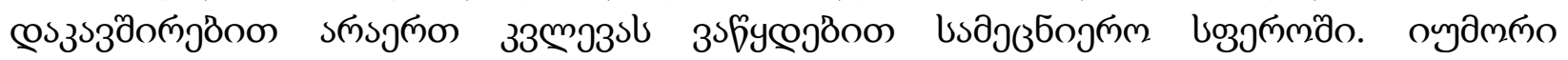

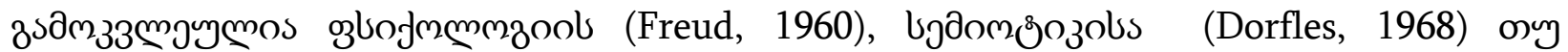

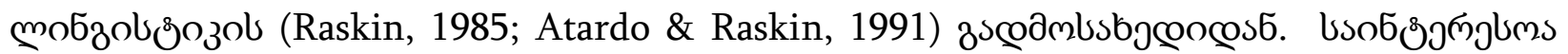

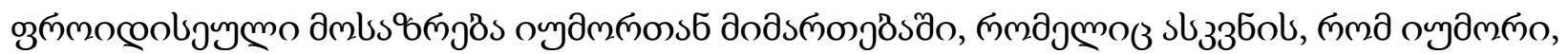

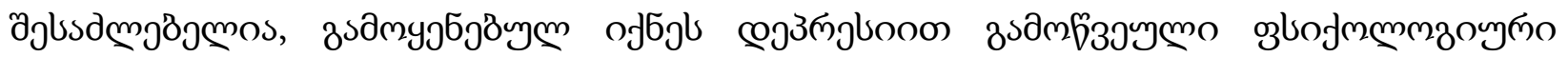

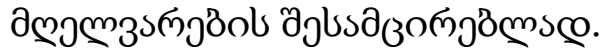




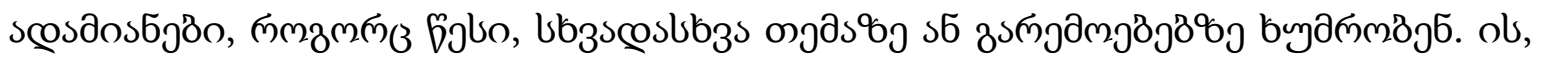

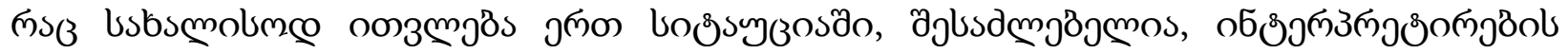

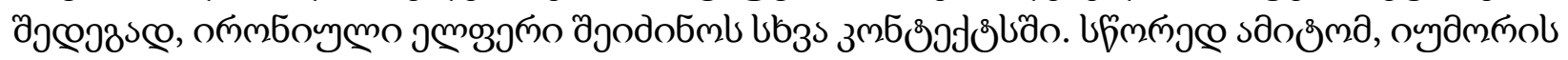

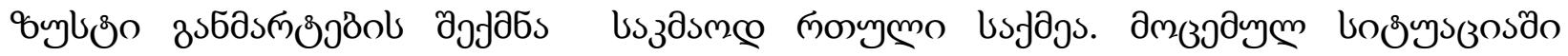

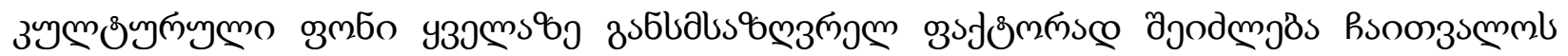

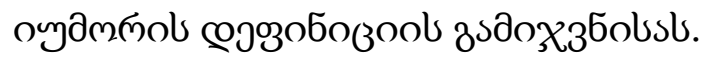

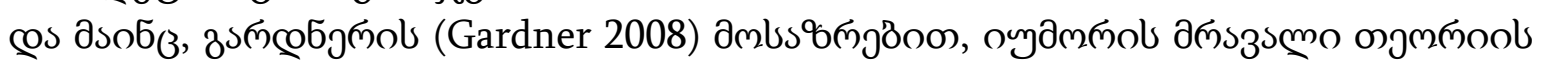

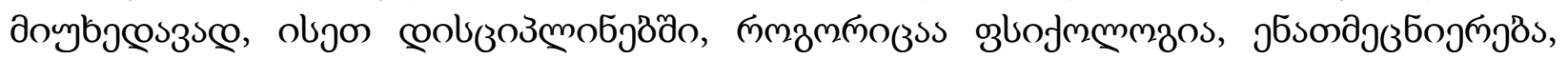

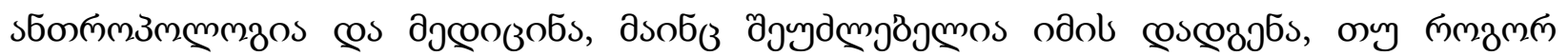

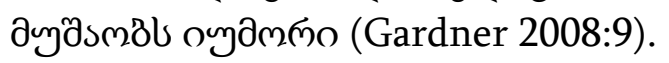

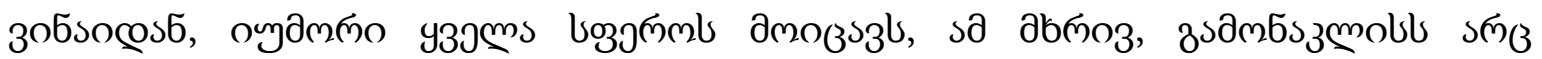

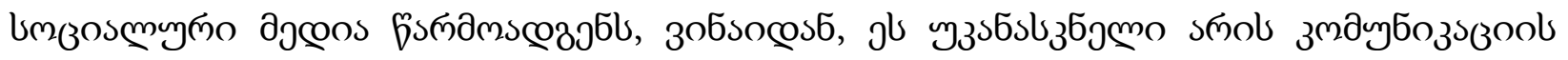

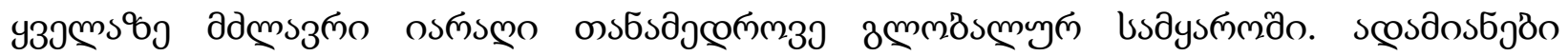

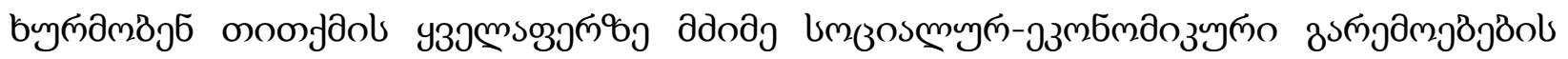

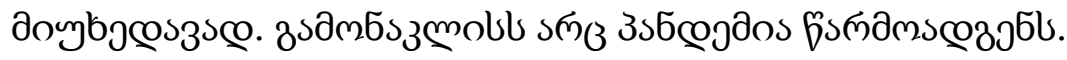

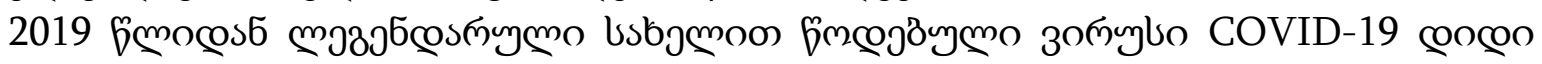

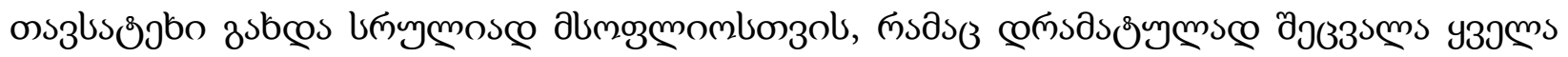

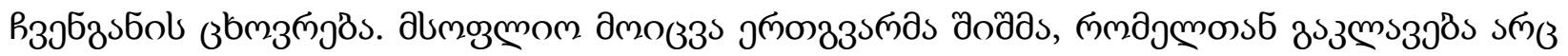

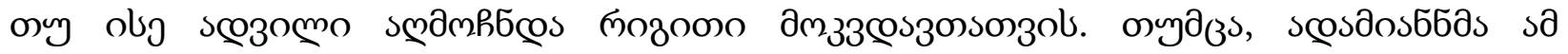

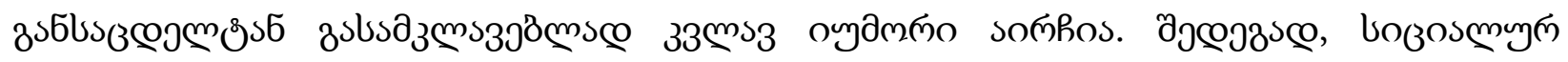

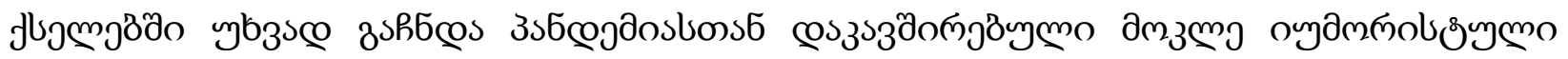

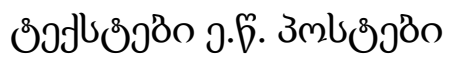

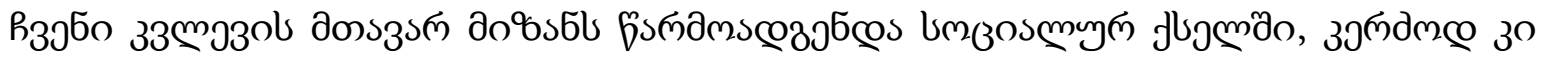
Facebook-

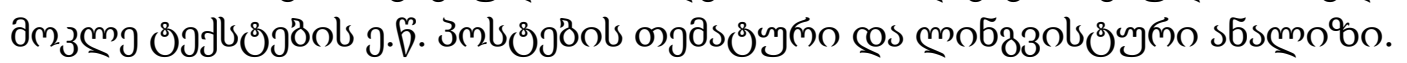

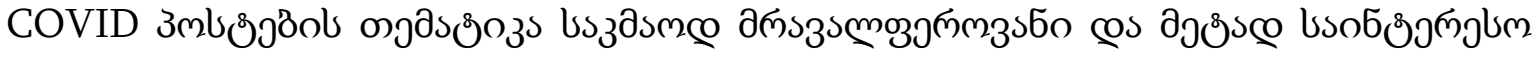

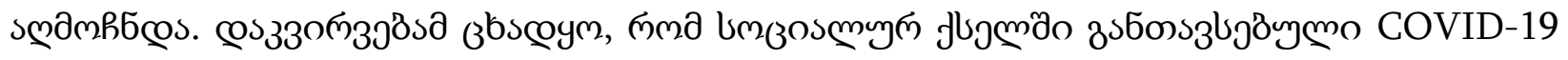

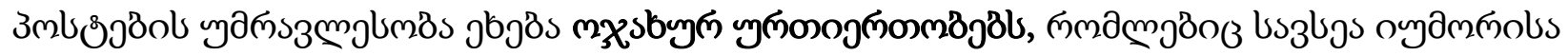

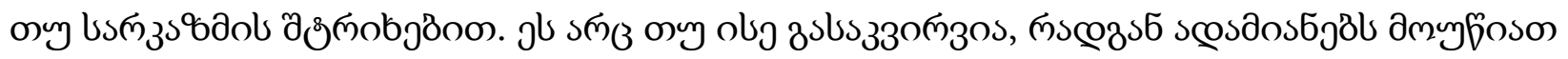

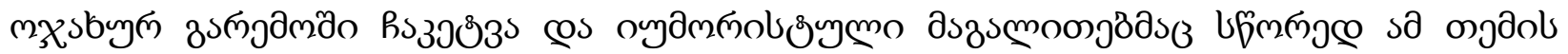

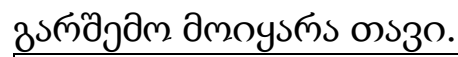

\begin{tabular}{|c|c|}
\hline \multicolumn{2}{|c|}{ 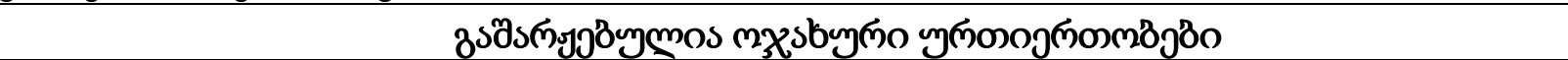 } \\
\hline 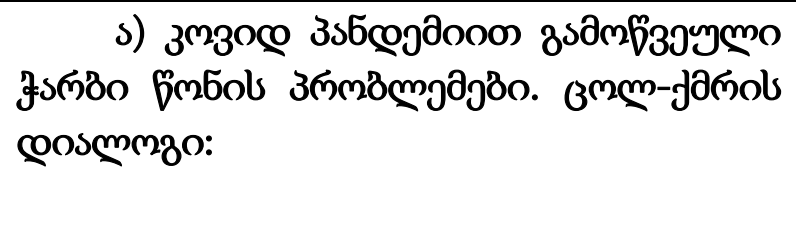 & 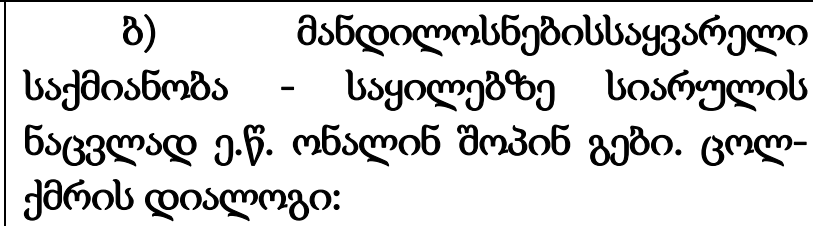 \\
\hline
\end{tabular}




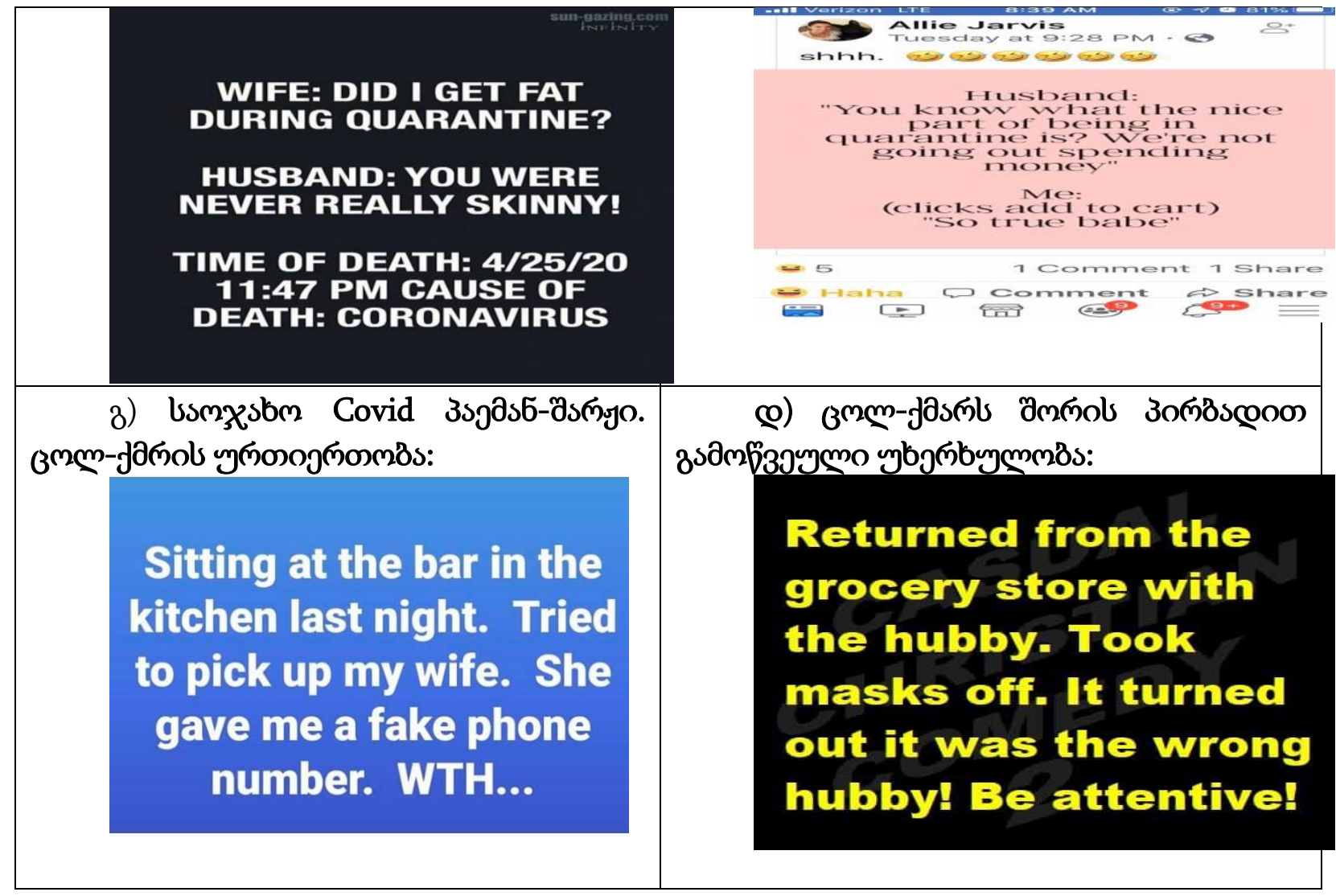

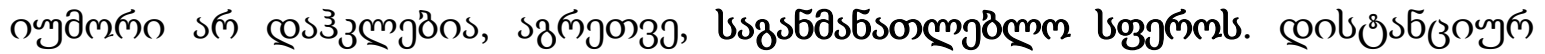

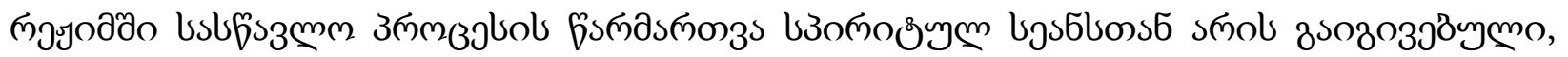

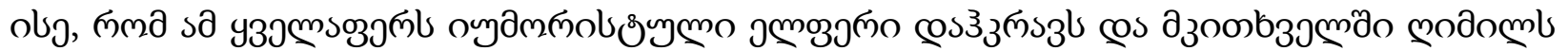
of333l: „There's someone who wants to join us." "Elizabeth, are you there?” "We can't hear

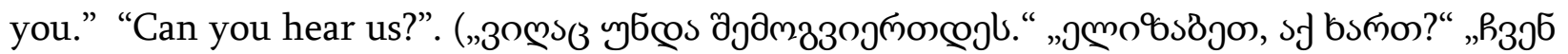

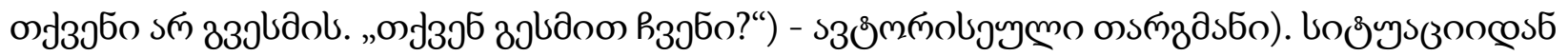

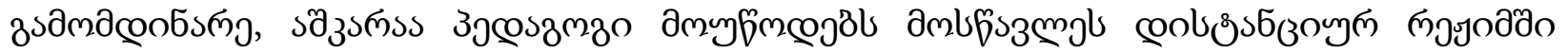

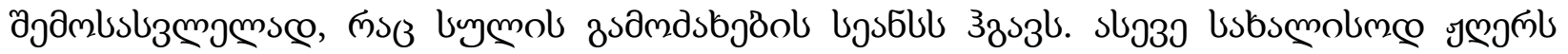

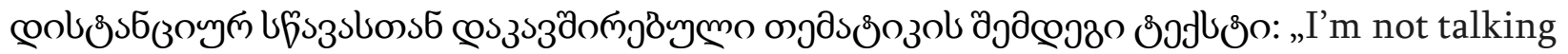

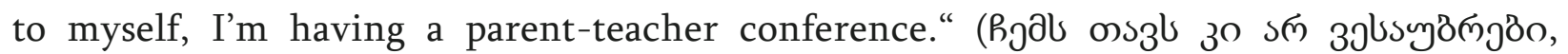

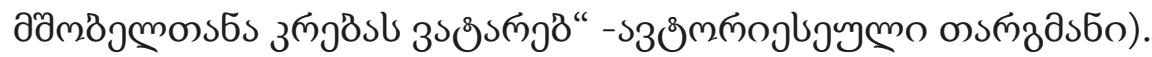

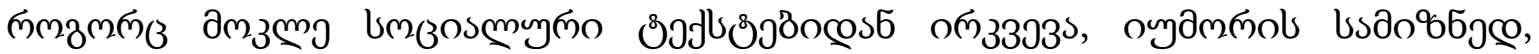

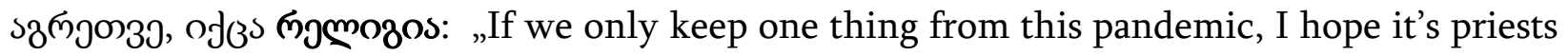

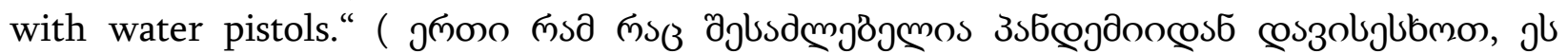

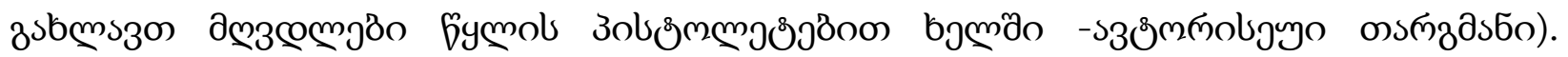

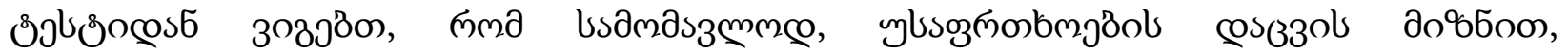

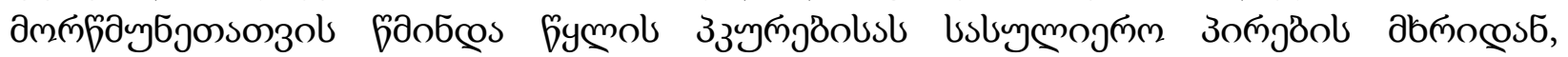

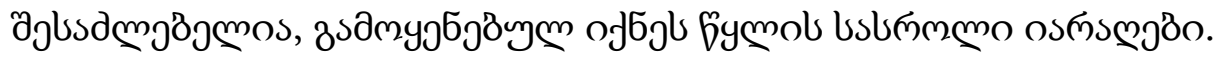

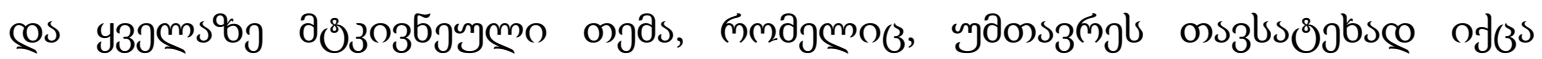

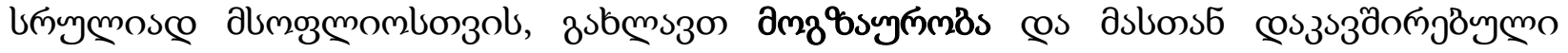




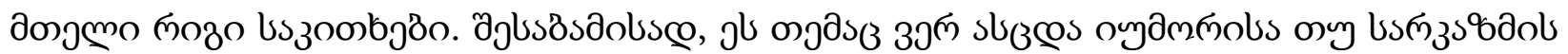

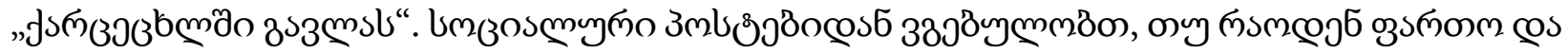

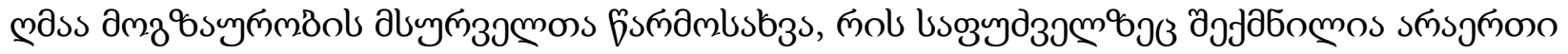

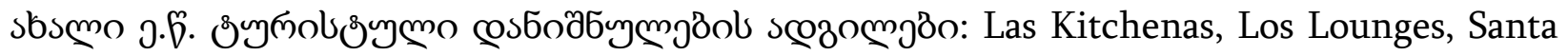
Bedrooms, Porto Gardenas, Los bed, Costa Del Balconia, St. Bathroom, La Rotonda De Sofa .....

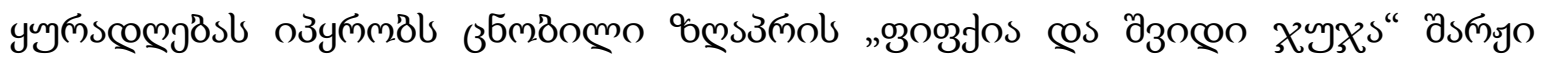

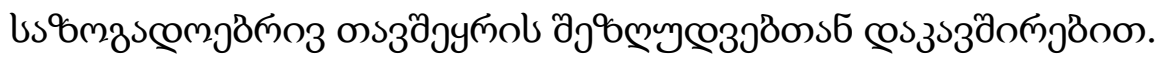

"In other news .... The Seven Dwarfs have been advised that as of today, they can only

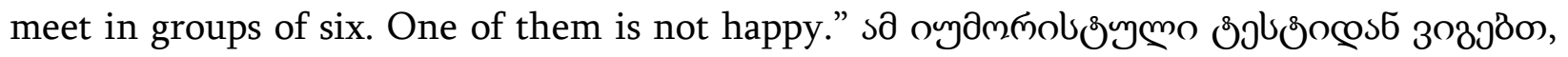

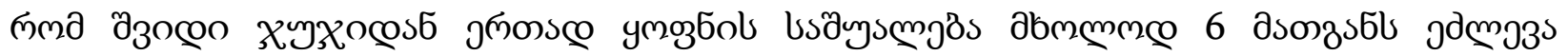

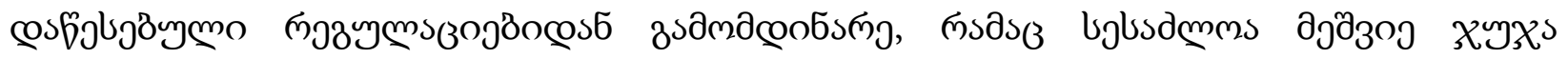

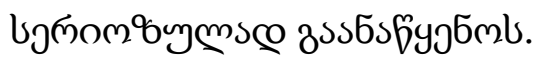

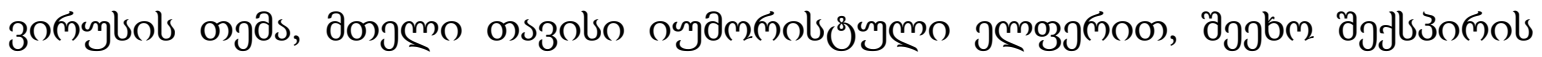

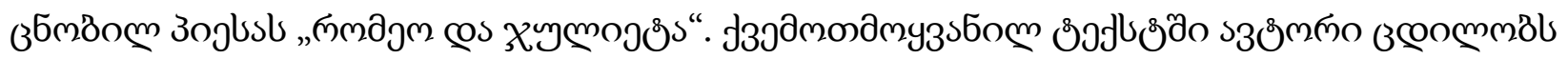

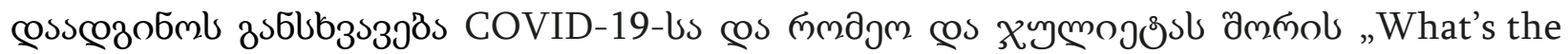
difference between COVID-19 and Romeo and Juliet? One's the coronavirus and the other

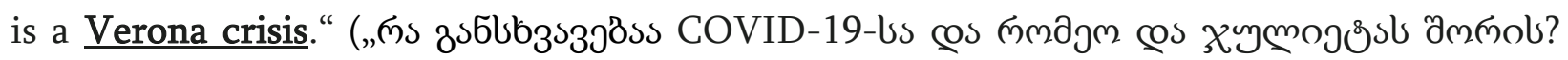

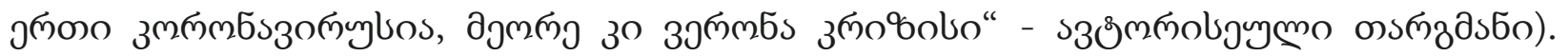

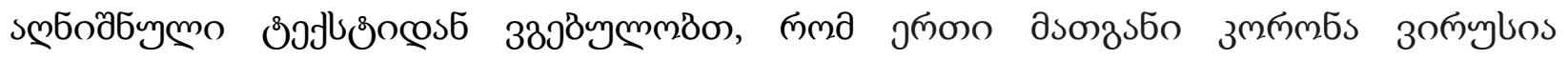

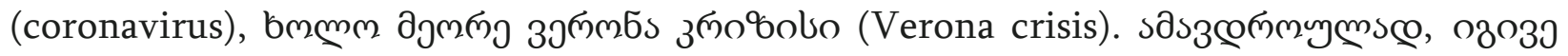

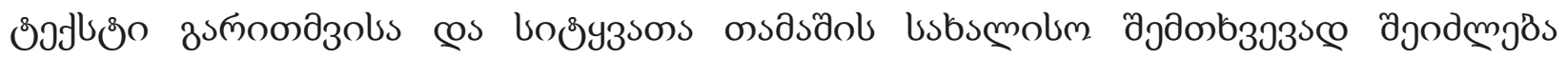

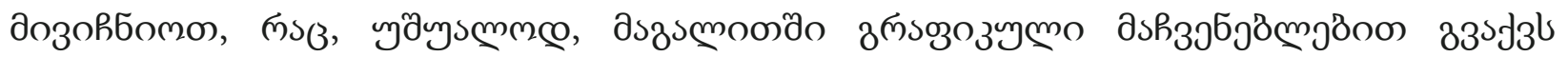
дободблдиммо.

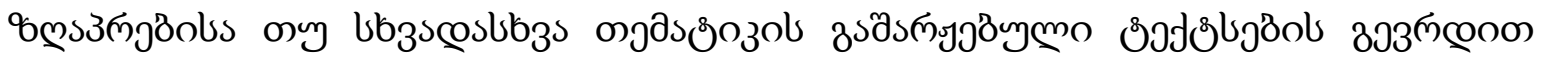

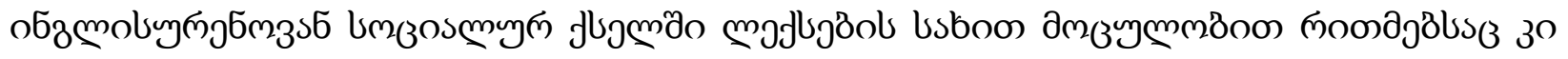

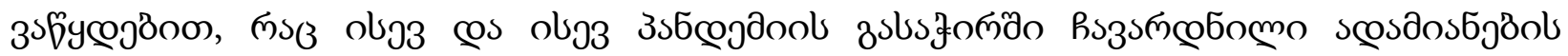

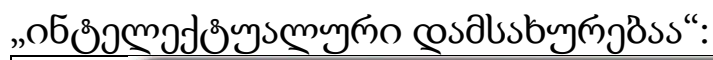

\begin{tabular}{|c|c|}
\hline 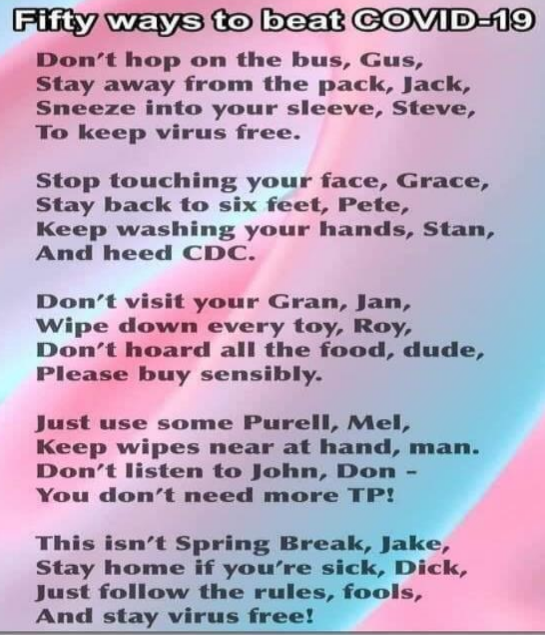 & $\begin{array}{l}\text { I do not want you in my house. } \\
\text { I do not want you or your spouse. } \\
\text { I do hot want to eat with you. } \\
\text { Seder One or Seder who. } \\
\text { Don't get me wrong. I think you're nice. } \\
\text { But the CDC gave out this advice. } \\
\text { rou must avoid one plague more } \\
\text { and shoo Elijah from gour door. } \\
\text { At next year's Seder. we will tell } \\
\text { how we were all saved by Purell. }\end{array}$ \\
\hline
\end{tabular}

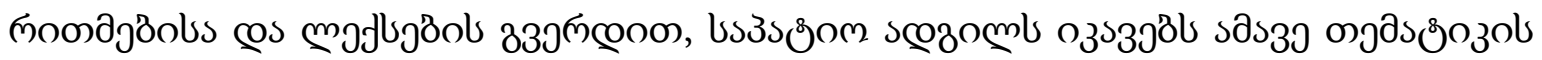

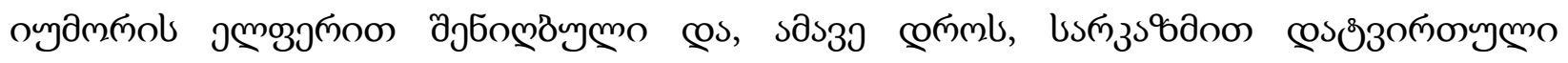




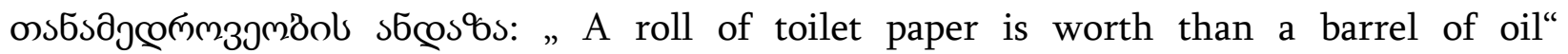

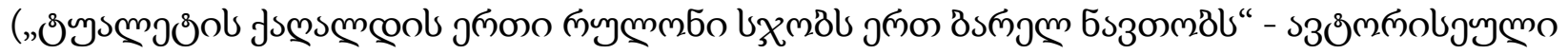
osm

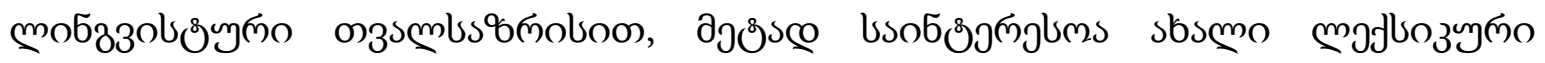

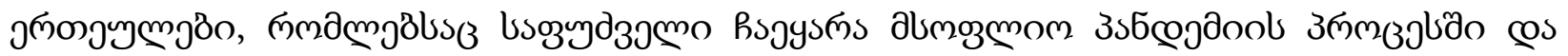

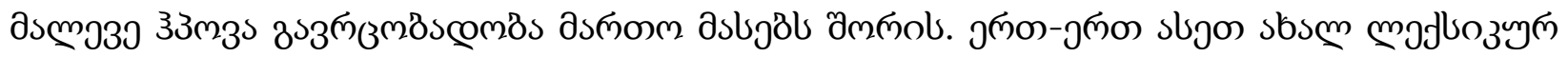

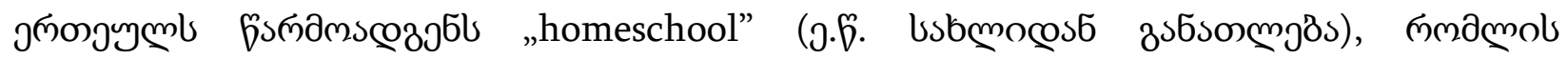

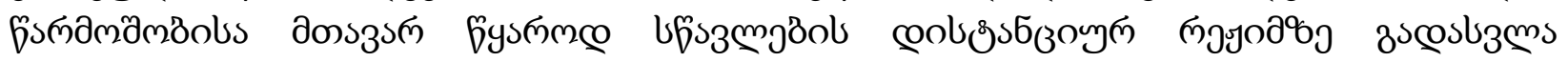

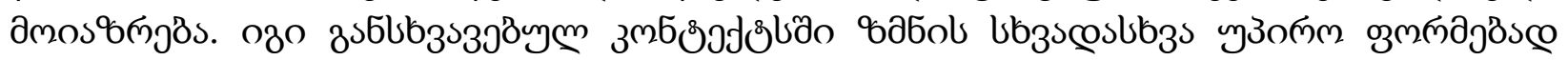
3393mобj8s. dş:

1) Stay at homeschooling tip \#102: Anyone who talks back or rolls eyes is now a janitor

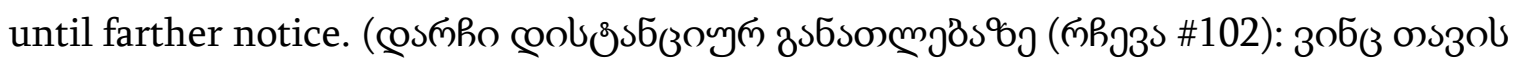

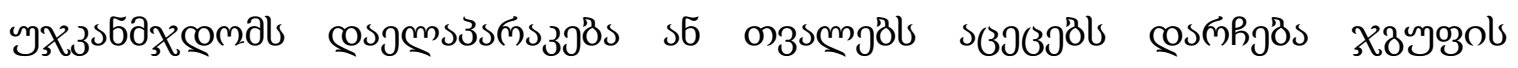

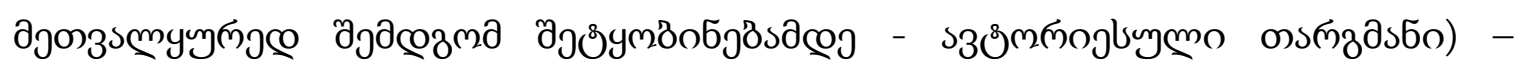
(зэмлуббеозо);

2) In 20 years our country will be run by people homeschooled by day drinkers. ( 20

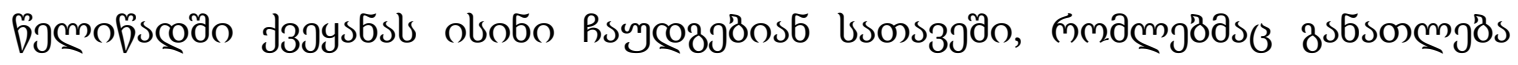

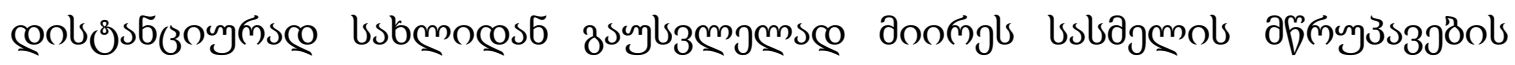

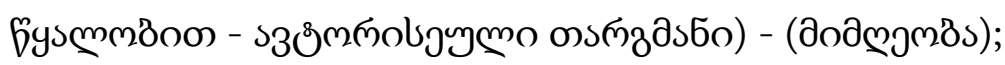

3) If you all don't start wearing masks then you are going to have to homeschool your kids

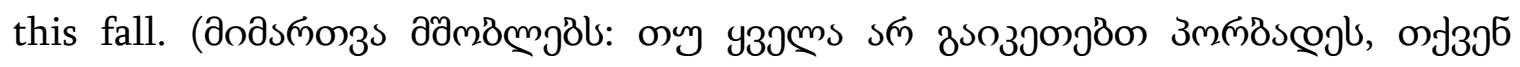

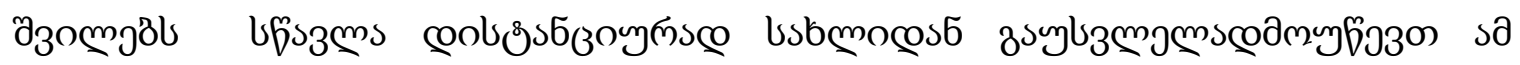

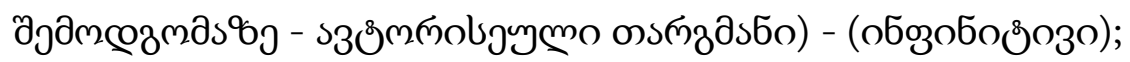

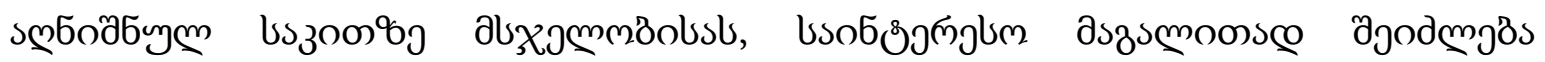

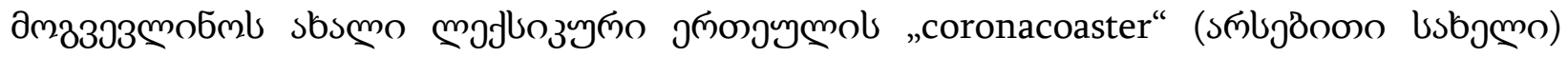

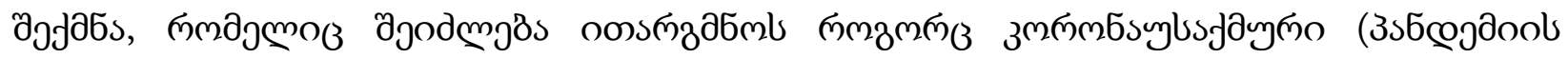
зјмом@до эls

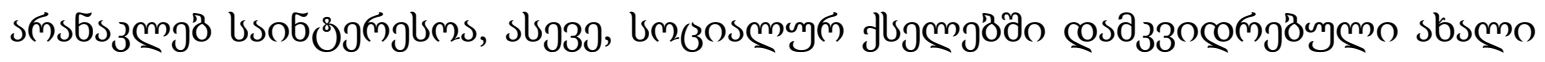

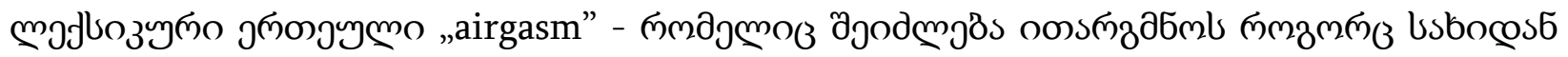

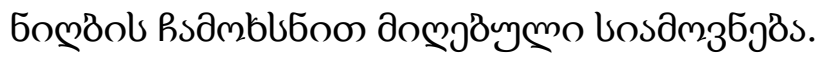

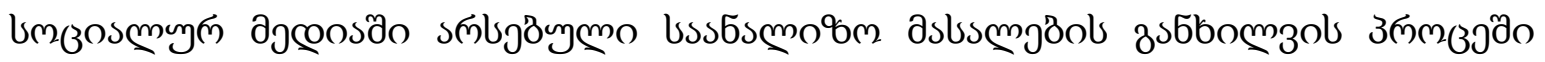

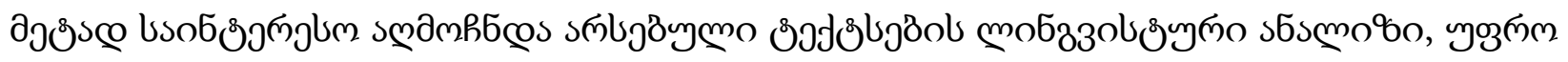

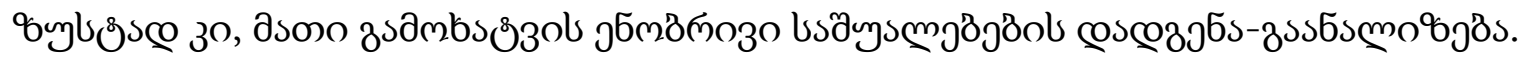

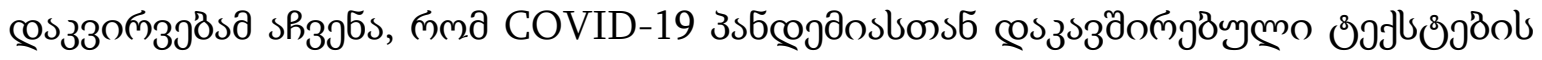

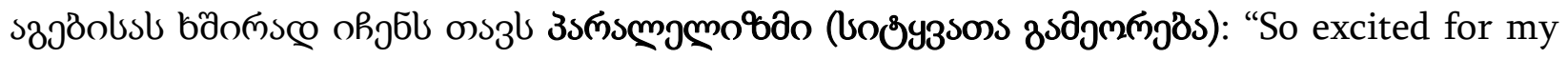
quarantine depression and my regular depression to meet my seasonal depression."

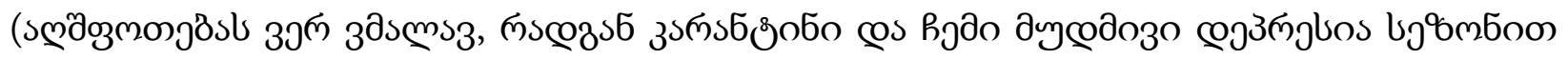

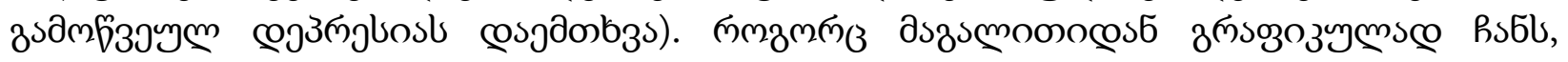




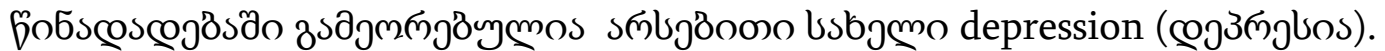

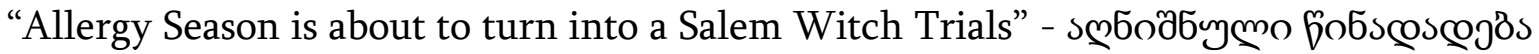

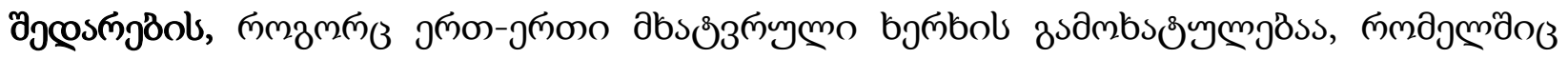

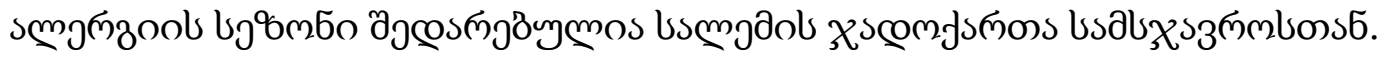

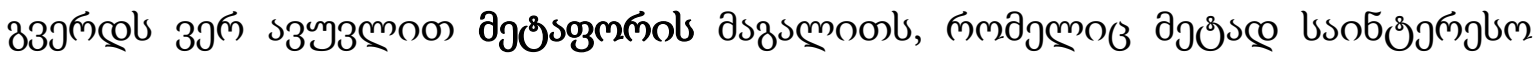

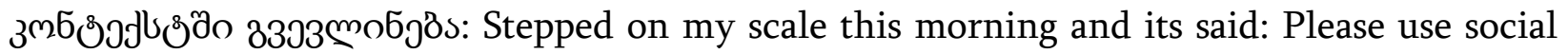

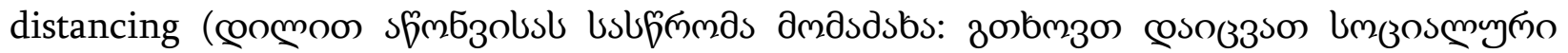

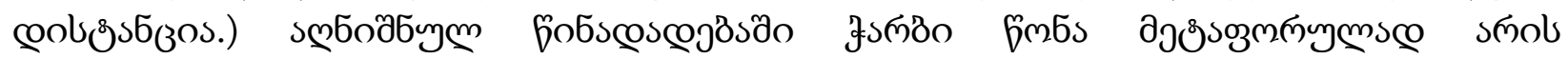

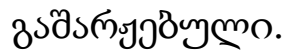

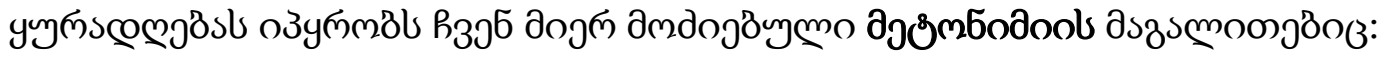

- Just heard the king of Spain has been quarantined on his private jet. That means the

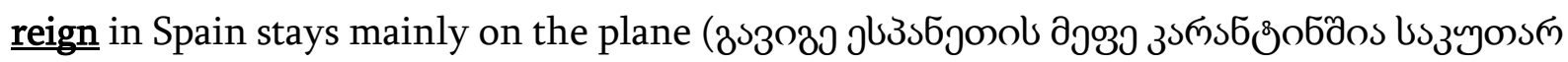

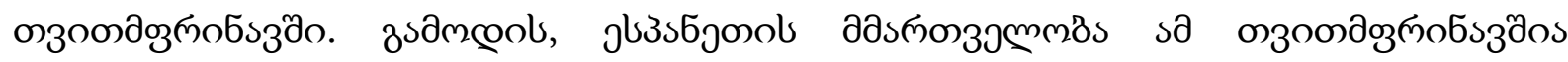
ams(зэymo.)

- $\quad$ I ran out of toilet paper and had to start using old newspapers. Times are rough.

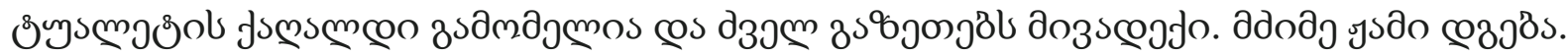

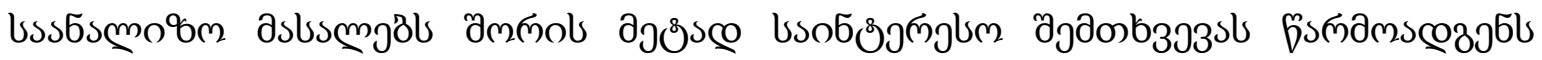

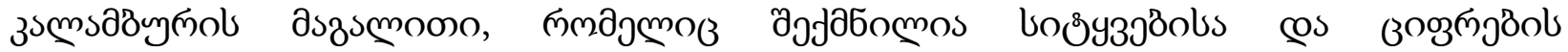

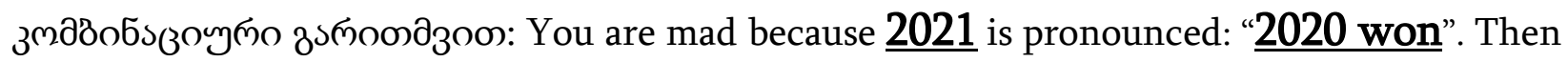
you realize that $\underline{2022}$ is $\mathbf{2 0 2 0 \text { too. }}$

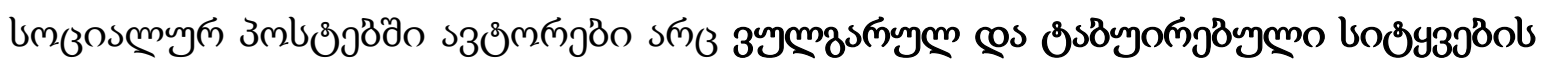

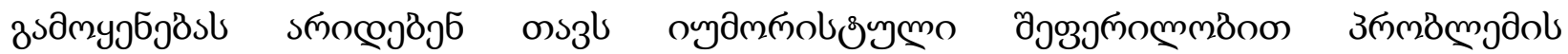

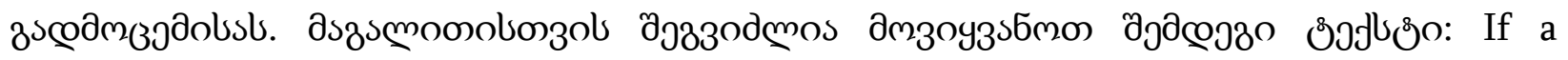
supervisor at work gets the covid do the people who kiss that ass need to be tested? (ony

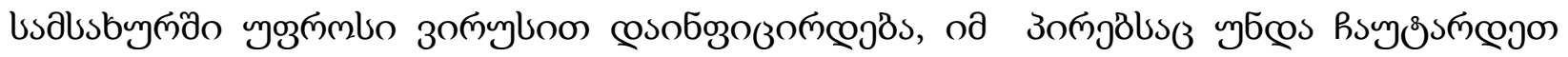

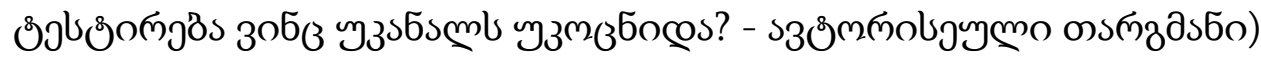

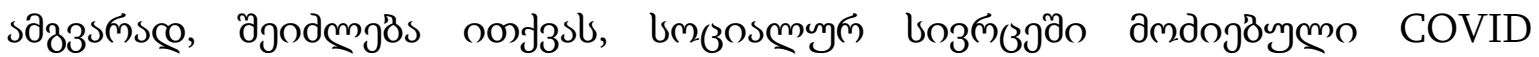

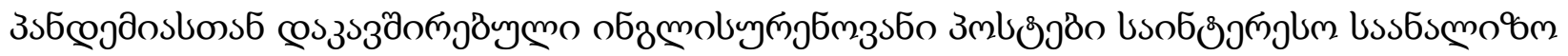

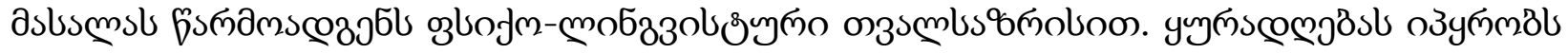
sа зmbతి

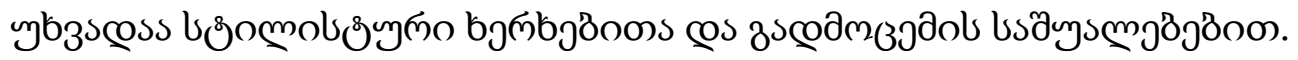

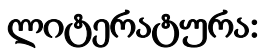

Attardo, S., and Raskin, V .1991. Script theory revisi(it)ed: Joke similarity and joke representation model Humor: International Journal of Humor Research, 4(3-4), 293- 347 
Dorfles, G. (1968). Kitsch: The World of Bad Taste. New York: Universe.

Freud, S. 1960. Jokes and their relation to the unconscious. London: Routledge \& Paul

Gardner, S. 2008 Three ways humor helps in the language classroom. The Language Teacher 32.6

Raskin, V. 1985. Semantic Mechanisms of Humor. Dordrecht: Reidel

\title{
For the Linguistic Conceptualization of Social Humor
}

\author{
Garibashvili Manana \\ Iakob Gogebashvili Telavi State University
}

\begin{abstract}
Humor plays an important role in people's lives, which helps them to communicate and share their feelings and thoughts among each other. Jokes and various humorous utterances are a form of communication that is constantly shared in the process of social interaction. That is why humor is, to some extent, recognized as a social phenomenon.

Since humor covers all areas, social media is no exception, as the latter is the most powerful tool of communication in the modern global world. People joke almost on everything despite difficult socio-economic circumstances and pandemic is no exception.

The main goal of our research was to observe and analyze the so-called pandemic-related posts on social networks, particularly Facebook and to make their thematic and linguistic analysis.

COVID 19 posts turned out to be quite diverse and very interesting. The observation has revealed that most of the posts on the social network concern the family relationships that are full of humor or sarcasm. This is not so surprising as people were forced to be locked in a family environment, That's why numerous examples of this type have been gathered around this very topic. Interesting materials on educational and travel topics were also found during the study.

Linguistic analysis of texts from social media, more precisely identification and analysis of the linguistic and literary means of expression, appeared to be very interesting.

The research has revealed that various stylistic means such as repetition (parallel constriction), metaphors, comparison/simile, metonymy etc. are frequently used for constructing COVID 19 texts in social media. Thus, the analyzed examples are very interesting material from a psycho-linguistic point of view.
\end{abstract}

Key words: Humor, social media, humorous utterance, Facebook, communication 